\title{
The Bochner curvature tensor on almost Hermitian manifolds
}

\author{
By L. VANHecke and D. JANSSENS \\ (Received June 22, 1977; Revised September 26, 1977)
}

\begin{abstract}
We prove a decomposition theorem for curvature tensors on a Hermitian vector space over $\boldsymbol{R}$ and use this to introduce Bochner curvature tensors. Applications which include the well known Kähler case are given for almost Hermitian manifolds.
\end{abstract}

\section{Introduction}

Singer and Thorpe [11] established a natural decomposition of curvature tensors on an $n$-dimensional real vector space with inner product and Nomizu [10] used this decomposition to study generalized curvature tensor fields. Kowalski [8] considered also a decomposition theory to study conformal differential geometry. In these papers the Weyl conformal curvature tensor is obtained in a very natural way as a projection of the Riemann curvature tensor.

Sitaramayya [12] and Mori [9] gave a similar decomposition to study curvature tensors on Kähler manifolds.

In this paper we extend these results, based on [14]. First we prove a decomposition theorem for a class of curvature tensors $L$ on a Hermitian vector space $V$ and derive the Bochner curvature tensor associated with $L$. Then we consider a large class of almost Hermitian manifolds and study some properties of the Bochner curvature tensor field associated with the Riemann curvature structure.

\section{Curvature tensors}

Let $V$ be an $n$-dimensional real vector space with inner product $g$. A tensor $L$ of type $(1,3)$ over $V$ is a bilinear mapping $L: V \times V \rightarrow \operatorname{Hom}(V, V)$ : $(x, y) \rightarrow L(x, y) . \quad L$ is called a curvature tensor on $V$ if it has the following properties :

AMS (MOS) Subject classifications (1970). Primary 53 B35, 53C55. Secondary 53 A 45.

Key words and phrases. Curvature tensors, decomposition theory, Bochner tensor, almost Hermitian manifolds. 
i) $L(x, y)=-L(y, x)$;

ii) $L(x, y)$ is a skew-symmetric endomorphism of $V$, i. e., $L(x, y, z, w)$ $+L(x, y, w, z)=0$ where $L(x, y, z, w)=g(L(x, y) z, w)$;

iii) $\subseteq L(x, y) z=0$, where $\mathfrak{S}$ denotes the cyclic sum over $x, y$ and $z$. This is the first Bianchi identity.

This means also that $L$ is a symmetric double form of type $(2,2)$ which satisfies the first Bianchi identity [2].

The Ricci tensor $L_{R}$ of type $(0,2)$ associated with $L$ is a symmetric bilinear function on $V \times V$ defined by

$$
L_{R}(x, y)=\operatorname{trace}(z \in V \mapsto L(z, x) y \in V) .
$$

Then, the Ricci tensor $Q=Q(L)$ of type $(1,1)$ is given by $L_{R}(x, y)=g(Q x, y)$ and the trace of $Q$ is called the scalar curvature $l=l(L)$ of $L$.

\section{2. $K_{i}$-curvature tensors}

Now let $V$ be a $2 n$-dimensional real vector space with a complex structure $J$ and a Hermitian product $g$, i. e.

$$
J^{2}=-I, g(J x, J y)=g(x, y)
$$

for all $x, y \in V, I$ denoting the identity transformation on $V$.

The Ricci $*$ tensor $L_{R}^{*}$ of type $(0,2)$ resp. $Q^{*}$ of type $(1,1)$ associated with a curvature tensor $L$ is defined by [5]

$$
L_{R}^{*}(x, y)=g\left(Q^{*} x, y\right)=\frac{1}{2} \operatorname{trace}(z \in V \mapsto L(x, J y) J z \in V) .
$$

$l^{*}=$ trace $Q^{*}$ is called the scalar $*$ curvature.

It follows from the theory of almost Hermitian manifolds [6], [7] that it is interesting to consider the following identities for a curvature tensor $L$ :

1) $L(x, y, z, w)=L(x, y, J z, J w)$;

2) $L(x, y, z, w)=L(J x, J y, z, w)+L(J x, y, J z, w)+L(J x, y, z, J w)$;

3) $L(x, y, z, w)=L(J x, J y, J z, J w)$.

$1)$ is called the Kähler identity. Further, let $\mathscr{K}_{i}(V)$ denote the vector space of all curvature tensors over $V$ satisfying the identity i).

Definition A $K_{i}$-curvature tensor $L$ on $V$ is a curvature tensor $L \in$ $\mathscr{K}_{i}(V)$. Then it is easy to check the following.

THEOREM 1.

i) $\mathscr{K}_{1}(V) \subset \mathscr{K}_{2}(V) \subset \cdot \mathscr{K}_{3}(V)$;

ii) $Q^{*}$ is a complex linear $\left(Q^{*} \circ J=J \circ Q^{*}\right)$ symmetric endomorphism of 
$V$ for any $L \in \mathscr{K}_{i}(V), i=1,2,3$. The same holds for $Q$.

We prove, for example, that $\mathscr{H}_{2}(V) \subset \mathscr{K}_{3}(V)$. Note that the second identity implies

$$
L(z, w, J x, J y)=L(J z, J w, J x, J y)-L(J z, w, x, J y)-L(J z, w, J x, y) .
$$

Substituting in 2) gives

$$
L(x, y, z, w)=L(J x, J y, J z, J w)+L(J x, y, z, J w)-L(x, J y, J z, w) .
$$

Hence we have also

$$
L(z, w, x, y)=L(J z, J w, J x, J y)+L(J z, w, x, J y)-L(z, J w, J x, y) .
$$

The required result follows now at once by adding the last two identities.

Finally we give an example of a $K_{i}$-curvature tensor which will play an important role in what follows. Let $x \wedge y$ be the skew-symmetric endomorphism of $V$ defined by $(x \wedge y) z=g(y, z) x-g(x, z) y$. Further we define $L_{A, B, \alpha}$ for any complex linear symmetric endomorphisms $A$ and $B$ of $V$ and any $\alpha \in R$, by

$$
\begin{aligned}
& L_{A, B, \alpha}(x, y)=A x \wedge B y+B x \wedge A y+J A x \wedge J B y+J B x \wedge J A y \\
& \quad+2 g(A x, J y) J B-2 g(J x, B y) J A+\alpha\{3 x \wedge y-J x \wedge J y-2 g(x, J y) J\} .
\end{aligned}
$$

It follows immediately that $L_{A, B, \alpha} \in \mathscr{H}_{2}(V)$ and $L_{A, B, \alpha} \in \mathscr{K}_{1}(V)$ if and only if $\alpha=0$.

We need also the following formulas which are easily verified:

$$
\begin{aligned}
& Q\left(L_{A, B, \alpha}\right)=A \operatorname{tr} B+B \operatorname{tr} A+2(A B+B A)+6 \alpha(n-1) I ; \\
& Q^{*}\left(L_{A, B, \alpha}\right)=Q\left(L_{A, B, \alpha}\right)-8 \alpha(n-1) I ; \\
& l\left(L_{A, B, \alpha}\right)=2 \operatorname{tr} A \operatorname{tr} B+4 \operatorname{tr} A B+12 \alpha n(n-1) ; \\
& l^{*}\left(L_{A, B, \alpha}\right)=l\left(L_{A, B, \alpha}\right)-16 \alpha n(n-1) .
\end{aligned}
$$

\section{Decomposition theorem}

In what follows we denote by $\mathcal{L}(V)$ the vector space of $K_{3}$-curvature tensors. This is a subspace of the tensor space of type $(1,3)$ over $V$ and has a natural inner product induced from that on $V$ :

$$
\langle L, \widetilde{L}\rangle=\operatorname{trace} \widetilde{L}^{T} \circ L=\sum_{i, j, k=1}^{2 n} g\left(L\left(e_{i}, e_{j}\right) e_{k}, \widetilde{L}\left(e_{i}, e_{j}\right) e_{k}\right),
$$

$\left\{e_{i}\right\}$ being an orthonormal basis of $V$. Further, let $\operatorname{Hom}_{c s}(V, V)$ denote the space of all complex linear symmetric endomorphisms of $V$ and define the 
Ricci contraction map $\mathscr{R}$ as follows :

$$
\mathscr{R}: \mathcal{L}(V) \rightarrow \operatorname{Hom}_{c s}(V, V) \times \boldsymbol{R}: L \mapsto\left(Q+3 Q^{*}, l-l^{*}\right) .
$$

Clearly $\mathscr{R}$ is linear. This is also the case for the map $h$ defined by

$$
h: \operatorname{Hom}_{c s}(V, V) \times \boldsymbol{R} \rightarrow \mathcal{L}(V):(A, \alpha) \mapsto L_{A, I, \alpha} .
$$

The definition of $\mathscr{R}$ and $h$ is based on geometrical considerations [14].

LemMa 1. $\mathscr{R}$ oh is an isomorphism.

Proof. It is sufficient to show that $\mathscr{R} \circ h$ is injective. Let $(A, \alpha) \in$ $\operatorname{Hom}_{c s}(V, V) \times \boldsymbol{R}$ with $\mathscr{R} h(A, \alpha)=0$. Hence $l\left(L_{A, I, \alpha}\right)=l^{*}\left(L_{A, I, \alpha}\right)$ and this implies with (1) that $\alpha=0$. Now, using $Q\left(L_{A, I, \alpha}\right)+3 Q^{*}\left(L_{A, I, \alpha}\right)=0$ we obtain from (1) $2(n+2) A+I \operatorname{tr} A=0$ which implies $\operatorname{tr} A=0$ and hence $A=0$.

Lemma 2. $\operatorname{Im} h$ is orthogonal to $\operatorname{Ker} \mathscr{R}$ in $\mathcal{L}(V)$.

PRoof. Let $A \in \operatorname{Hom}_{c s}(V, V), L=h(A, \alpha)=L_{A, I, \alpha}$ and $\widetilde{L} \in \operatorname{Ker} \mathscr{R}$. It is always possible to choose an orthonormal $J$-basis $\left\{e_{i}, J e_{i}, i=1,2, \cdots, n\right\}$ such that $A e_{i}=\lambda_{i} e_{i}, A J e_{i}=\lambda_{i} J e_{i}$. Further we have $\tilde{l}=\tilde{l}^{*}=0$. The orthogonality follows now by a straightforward calculation using the first Bianchi identity for $\widetilde{L}$.

Lemma 3. $\operatorname{dim} \operatorname{Im} h+\operatorname{dim} \operatorname{Ker} \mathscr{R}=\operatorname{dim} \mathcal{L}(V)$.

Proof. It follows from Lemma 1 that $h$ is injective and $\mathscr{R}$ is surjective. Hence $\operatorname{dim} \operatorname{Im} h=\operatorname{dim} \operatorname{Hom}_{c s}(V, V) \times \boldsymbol{R}=\operatorname{dim} \mathcal{L}(V)-\operatorname{dim} \operatorname{Ker} \mathscr{R}$.

Putting $\mathcal{L}_{\boldsymbol{B}}(V)=\operatorname{Ker} \mathscr{R}$ we have

LEMMA 4. $\mathcal{L}(V)=\operatorname{Im} h \oplus \mathcal{L}_{B}(V)$.

Now let $L \in \mathcal{L}(V)$. Using Lemma 4 we have

$$
L=h(A, \alpha)+L_{B}, A \in \operatorname{Hom}_{c s}(V, V), \alpha \in \boldsymbol{R}, L_{B} \in \mathcal{L}_{B}(V) .
$$

Define the map $\mathscr{D}: \mathcal{L}(V) \rightarrow \operatorname{Hom}_{c s}(V, V) \times \boldsymbol{R}$ by $\mathscr{Q} L=(A, \alpha)$. We call $\mathscr{Z}$ the deviation map (see also [8]). Hence we have if $j$ denotes the canonical inclusion of $\mathcal{L}_{B}(V)$ in $\mathcal{L}(V)$ :

Decomposition Theorem. There is a unique linear map $\mathscr{B}: \mathcal{L}(V) \rightarrow$ $\mathcal{L}_{B}(V)$, called the Bochner map, and a unique linear map $\mathscr{D}: \mathcal{L}(V) \rightarrow \mathrm{Hom}_{\mathrm{cs}}$ $(V, V) \times \boldsymbol{R}$, called the deviation map, such that the following commutative diagram with two exact sequences holds: 


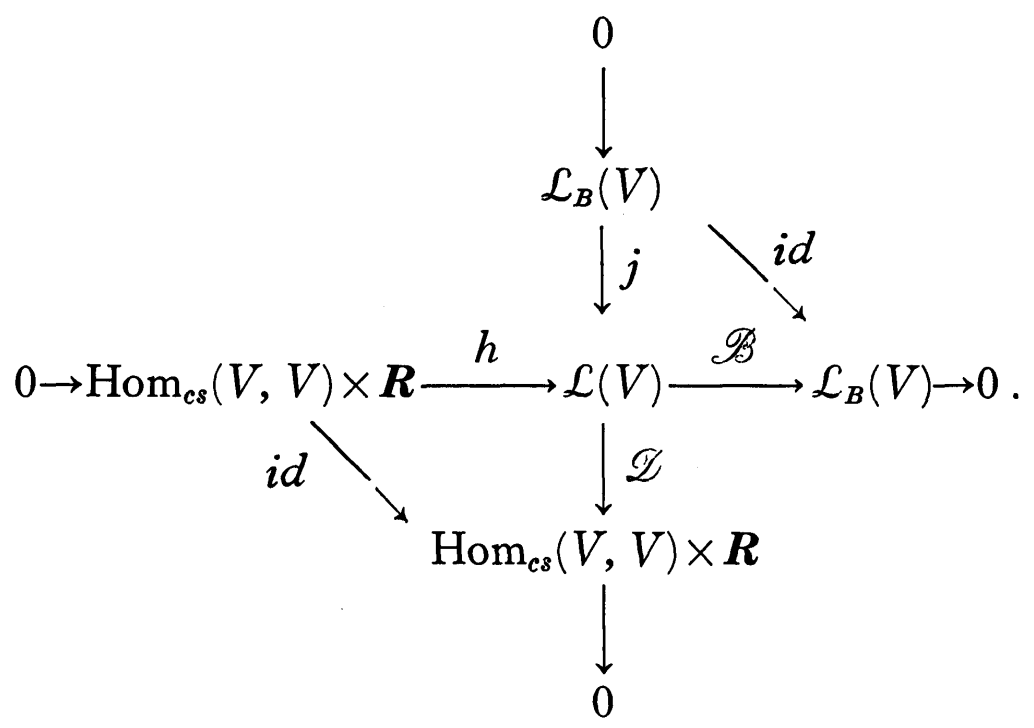

Moreover, the decomposition $\mathcal{L}(V)=\operatorname{Im} h \oplus \mathcal{L}_{B}(V)$ is orthogonal and hence the Bochner map $\mathscr{B}$ is the orthogonal projection of $\mathcal{L}(V)$ onto its subspace $\mathcal{L}_{B}(V)$.

It is possible to express the maps $\mathscr{B}$ and $\mathscr{D}$ explicitly. Indeed we have Theorem 2. Let $L \in \mathcal{L}(V)$. Then

$$
\begin{aligned}
& \mathscr{D} L=(A, \alpha), \\
& \mathscr{B} L=L-h \mathscr{D} L=L-L_{A, I, \alpha}
\end{aligned}
$$

where

$$
A=\frac{1}{8(n+2)}\left(Q+3 Q^{*}-\frac{l+3 l^{*}}{4(n+1)} I\right), \quad \alpha=\frac{1}{16 n(n-1)}\left(l-l^{*}\right) .
$$

PRoof. It follows from (2) that $l-l^{*}=l\left(L_{A, I, \alpha}\right)-l^{*}\left(L_{A, I, \alpha}\right)$. This gives the required expression for $\alpha$. Further we have $Q+3 Q^{*}=Q\left(L_{A, I, \alpha}\right)+3 Q^{*}$ $\left(L_{A, I, \alpha}\right)$ and this implies $8(n+2) A=Q+3 Q^{*}-4 I \operatorname{tr} A$. Taking the trace we get $16(n+1) \operatorname{tr} A=l+3 l^{*}$ and this gives the formula for $A$.

Definition [14]. Curvature tensors belonging to $\mathcal{L}_{B}(V)$ are called Bochner curvature tensors and the tensor $L_{B}$ is called the Bochner curvature tensor associated with $L \in \mathcal{L}(V)$.

\section{Applications}

Let $M$ be a Riemannian manifold with metric tensor $g$ and Riemannian connection $\nabla$. For each point $m \in M$ we may consider curvature tensors $L$ over the tangent space $T_{m}(M)$ with inner product $g_{m}$. A differentiable curvature tensor field $L$ on $M$ is called a generalized curvature tensor field [10]. We recall that $L$ is proper [10] or a Riemannian double form of 
type $(2,2)$ [2] if it satisfies the second Bianchi identity, that is $\mathfrak{S}\left(\nabla_{X} L\right)(Y, Z)$ $=0$ for all $X, Y, Z \in \mathscr{X}(M)$, where $\mathscr{X}(M)$ denotes the Lie algebra of $C^{\infty}$ vector fields on $M$.

Now, let $M$ be an almost Hermitian manifold, that is, the tangent bundle has an almost complex structure $J$ and a Riemannian metric $g$ such that $g(J X, J Y)=g(X, Y)$ for all $X, Y \in \mathscr{Z}(M)$. In the same way as before we may define generalized $K_{i}$-curvature tensor fields.

In what follows we take for $L$ the Riemann-Christoffel curvature tensor $R$. This is a proper tensor field and we suppose that $R_{m} \in \mathcal{L}(V), V=T_{m} M$. Then we find that the associated Bochner tensor $R_{B}=B$ is the well known Bochner tensor if $M$ is a Kähler manifold $(\nabla J=0)$. For such a manifold we know that $M$ is a complex space form if and only if $M$ is Bochner flat $(B=0)$ and Einsteinian.

In order to state a generalization we say that a curvature tensor $L$ is Einsteinian resp. *Einsteinian if $Q=\lambda I$ resp. $Q^{*}=\lambda^{*} I$ and we recall that an almost Hermitian manifold with $R \in \mathcal{L}(V)$ is a generalized complex space form [13] if an only if $R=L_{\frac{\mu}{8} I, I, \frac{\alpha}{4}}$, i. e.

$$
R(X, Y)=\frac{\mu+3 \alpha}{4} X \wedge Y+\frac{\mu-\alpha}{4}\{J X \wedge J Y+2 g(X, J Y) J\}
$$

$\mu$ is the holomorphic sectional curvature and $\frac{1}{4}(\mu+3 \alpha)$ the antiholomorphic sectional curvature. Both curvatures are pointwise constant. Further, a nearly Kähler manifold $M$ is an almost Hermitian manifold such that $\left(\nabla_{X} J\right) X=0$ for all $X \in \mathscr{X}(M)$ [3]. It follows then that $S^{6}$ is a generalized complex space form with respect to the three nearly Kähler structures on $S^{6}$ [1]. We have also that for any nearly Kähler manifold $R \in \mathcal{L}(V)$ [3]. So we obtain

THEOREM 3. Let $M$ be an almost Hermitian manifold with $R \in \mathcal{L}(V)$. Then $M$ is a generalized complex space form if and only if it is a Bochner flat Einstein and $*$ Einstein manifold.

Finally, using a classification theorem of A. Gray [4], we have

THEOREM 4. Let $M^{n}$ be a nearly Kähler manifold with complex dimension $n>2$. Then $M^{n}$ is a Bochner flat Einstein and $*$ Einstein manifold if and only if it is locally isometric to a complex space from $\left(C^{n}, C P^{n}(\mu)\right.$ or $\left.C D^{n}(\mu)\right)$ or $S^{6}(\mu)$, $\mu$ denoting the holomorphic sectional curvature.

\section{Remarks}

a. Suppose $M^{n}$ is almost Hermitian and $R \in \mathcal{L}(V)$. One finds that the 
properties for $B$ in relation with the theory of submanifolds are analogous with those in the Kähler case (see for example [14]).

b. The given decomposition method applies also to derive the well known concircular and projective curvature tensors as projections of the Riemann tensor. This is also true for the complex analogous tensors on an almost Hermitian manifold. Moreover, one may introduce in the same way a Bochner curvature tensor on a class of almost contact metric manifolds which includes for example the Sasakian, nearly Sasakian and normal cosymplectic manifolds. This will be shown in another paper.

\section{References}

[1] A. Gray: Almost complex submanifolds of the six sphere, Proc. Amer. Math. Soc. 20 (1969), 277-279.

[2] A. GRAY: Some relations between curvature and characteristic classes, Math. Ann. 184 (1970), 257-267.

[3] A. Gray: Nearly Kähler manifolds, J. Diff. Geom. 4 (1970), 283-309.

[4] A. GRAY: Classification des variétés approximativement kählériennes de courbure sectionnelle holomorphe constante, C. R. Acad. Sc. Paris A 279 (1974), 797-800.

[5] A. GRAY: The structure of nearly Kähler manifolds, Math. Ann 223 (1976), 233248.

[6] A. GRAY: Curvature identities for Hermitian and almost Hermitian manifolds, Tôhoku Math. J. 28 (1976), 601-612.

[7] A. GRAY and L. VANHECKE: Almost Hermitian manifolds with constant holomorphic sectional curvature, to appear.

[8] O. KOWALSKI: Partial curvature structures and conformal geometry of submanifolds, J. Diff. Geom. 8 (1973), 53-70.

[9] H. MORI : On the decomposition of generalized K-curvature tensor fields, Tôhoku Math. J. 25 (1973), 225-235.

[10] K. NomizU: On the decomposition of generalized curvature tensor fields, Differential Geometry (in honour of K. Yano), Kinokuniya, Tokyo, 1972, 335-345.

[11] I. M. SINGER and J. A. THORPE: The curvature of 4-dimensional Einstein spaces, Global Analysis (papers in honour of K. Kodaira), University of Tokyo Press, Tokyo, 1969, 355-365.

[12] M. Sitaramayya : Curvature tensors in Kaehler manifolds, Trans. Amer. Math. Soc. 183 (1973), 341-351.

[13] L. VANHECKE: Antiholomorphic submanifolds of generalized complex space forms, Bull. Inst. Math. Acad. Sinica 4 (1976), 127-140.

[14] L. VANHECKE: On the decomposition of curvature tensor fields on almost Hermitian manifolds, Differential Geometry, Proc. Conference, Dep. of Math., Michigan State Univ., East Lansing, 1976, 16-33.

Department of Mathematics, Katholieke Universiteit Leuven, Celestijnenlaan 200 B, B-3030 Heverlee, Belgium. 